\title{
Design Concept for 100kV-Level, 1.5-cell, RF Photo-Guns
}

W. Andreas Schroeder,* Joel A. Berger,* and John G. Power**

* University of Illinois at Chicago, Department of Physics (m/c 273), 845 W. Taylor Street (rm. 2236), Chicago, IL 60607-7059

** Advanced Accelerator R\&D Group, High Energy Physics Division, Argonne National Laboratory, Argonne, IL 60439

Ultrafast electron microscopy (UEM) aims to combine the high spatial (sub-nm) resolution of electron microscopy with the high temporal (sub-ps) resolution afforded by today's ultrashort pulse lasers [1]. In order to attain the desired high time resolution at the specimen, temporal electron pulse broadening due to both the intrinsic dispersion (i.e., a finite non-zero energy width) and intra-pulse space charge effects must be reversed. For the necessary electron pulse compression, the use of a RF cavity after a 100kV-level DC photo-gun has been proposed [2], and recently demonstrated [3], for ultrafast electron diffraction (UED) experiments. A potential alternative to such two component systems is a RF photo-gun in which electron acceleration and pulse compression can be combined in a single device. However, RF photo-guns generally operate at relativistic (a few MeV) electron energies, where electron optics for microscopy have not been developed and the small electron wavelength thus implies large post-specimen distances for UED measurements [4,5].

Here we present a design concept for a 100kV-level RF photo-gun suitable for both UED and UEM applications. The RF gun borrows the same basic 1.5-cell geometry of MeV-level relativistic photoguns [4,5], but the cell length and RF field amplitude are reduced to allow for operation at $\sim 100 \mathrm{kV}$. Figure 1 shows the performance characteristics of a $1.3 \mathrm{GHz}$ (L-band) $100 \mathrm{kV}$ RF gun with a $34 \mathrm{~mm}$ cell length. A contour plot of the exiting electron energy as a function of the RF phase $\left(\phi_{\mathrm{RF}}\right)$ at photoelectron generation and the RF field amplitude (Figure 1(a)) indicates that this gun geometry is capable of operation in the range $\sim 90-150 \mathrm{kV}$. Reduction of the cell length allows for lower energy operation (e.g., a $25 \mathrm{~mm}$ cell length for $\sim 50 \mathrm{kV}$ ), while longer cell lengths provide higher (relativistic) electron energies. Analysis of the RF field supported by the $100 \mathrm{kV}$ gun using SUPERFISH produces the characteristic sinusoidal axial field amplitude of a 1.5-cell RF gun (Figure 1(b)). Figure 1(c) shows the performance of the $100 \mathrm{kV}$ RF photo-gun for a RF field amplitude of $3.1 \mathrm{MV} / \mathrm{m}$ and $\phi_{\mathrm{RF}}=$ $0.27 \pi\left(49^{\circ}\right)$ - conditions under which a temporal focus (minimum longitudinal length) of the electron pulse is generated at a distance of $30 \mathrm{~cm}$ after the gun. The latter simulation of the electron pulse propagation dynamics, using an extended analytical Gaussian (AG) model [6], also indicates that the minimum duration of the compressed electron pulse can be more than 100 times less than the duration of the laser pulse incident on the gun's photocathode [7,8]. Simulations of the RF gun performance using PARMELA (a particle tracking (GPT-like) code) are consistent with results obtained using the less computationally intensive AG model.

The effect of intra-pulse space-charge on the temporal pulse compression fidelity and the required $\mathrm{RF}$ drive power levels to operate the RF photo-gun will be discussed. The performance and relative merits of the 1.5-cell RF photo-gun will also be compared to two other compact 100kV-level photogun technologies capable of providing temporal pulse compression: (i) the single-cell 'pill-box' RF photo-gun proposed by E. Fill et al. [7], and (ii) the hybrid DC-AC photo-gun (i.e., a RF pulse compression cavity directly after a DC photo-gun) analyzed by L. Veisz, et al. [8]. 


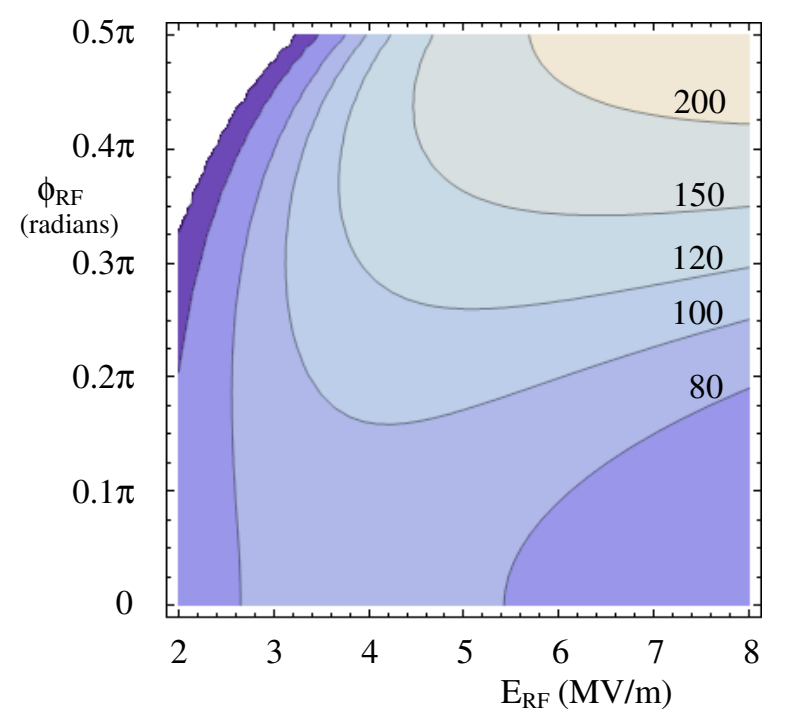

(a)

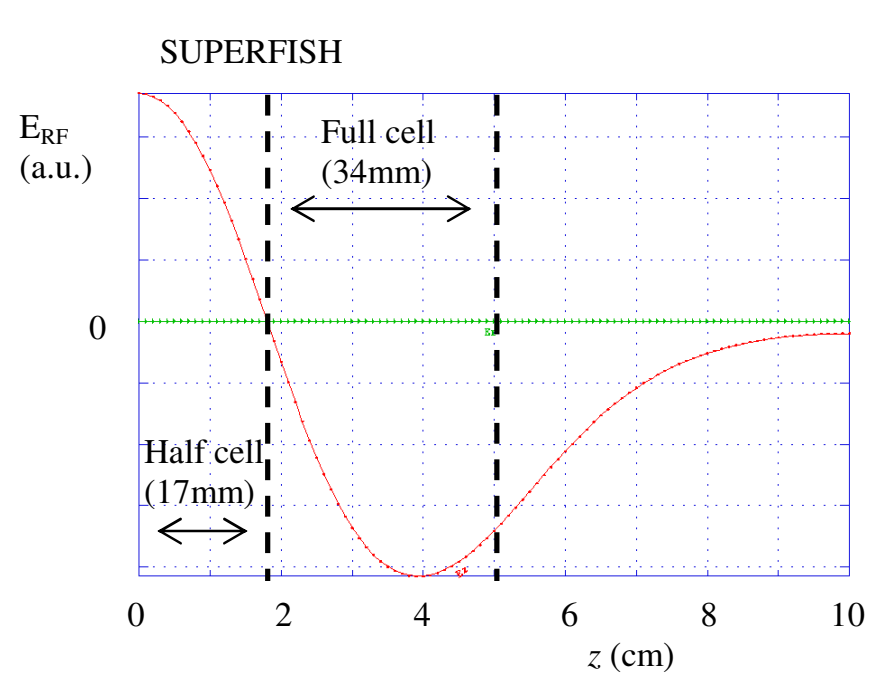

(b)

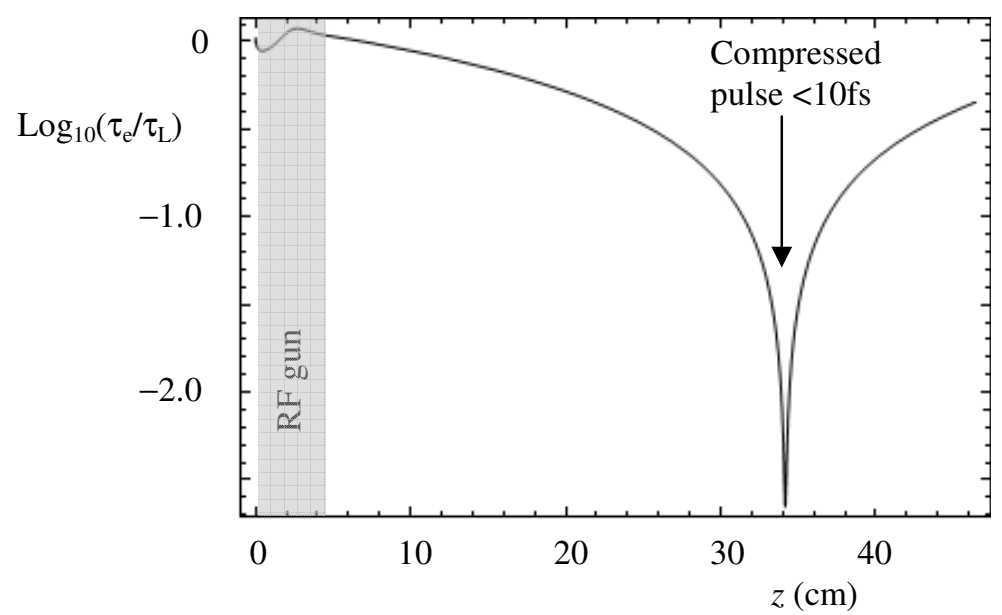

Simulation conditions:

Ta photocathode:

Work function, $\Phi=4.25 \mathrm{eV}$

Fermi energy, $\varepsilon_{\mathrm{F}}=5.3 \mathrm{eV}$

Laser radiation source:

Pulse duration, $\tau_{\mathrm{L}}=1 \mathrm{ps}$

Photon energy, $\hbar \omega=4.75 \mathrm{eV}$

Space-charge effects

neglected

(c)

FIG 1. (a) Contour plot of the exiting electron energy (labeled in $\mathrm{kV}$ ) as a function of the RF phase $\left(\phi_{\mathrm{RF}}\right)$ at photoelectron generation and the RF field amplitude. (b) SUPERFISH evaluation of the axial RF field amplitude in the 1.5-cell photo-gun with a cell length of $34 \mathrm{~mm}$. (c) Ratio of the electron pulse duration $\left(\tau_{\mathrm{e}}\right)$ to the laser pulse duration $\left(\tau_{\mathrm{L}}\right)$ for $\mathrm{E}_{\mathrm{RF}}=3.1 \mathrm{MV} / \mathrm{m}$ and $\phi_{\mathrm{RF}}=0.27 \pi$.

\section{References}

[1] W.E. King, et al., J. Appl. Phys. 97 (2005) 111101.

[2] T. van Oudheusen, et al., J. Appl. Phys. 102 (2007) 093501.

[3] T. van Oudheusden, et al., Phys. Rev. Lett. 105 (2010) 264801.

[4] J.B. Hastings, et al., Appl. Phys. Lett. 89 (2006) 184109.

[5] P. Musumeci, J.T. Moody, and C.M. Scoby, Ultramicroscopy 108 (2008) 1450.

[6] W.A. Schroeder and J.A. Berger, J.Appl. Phys. 108 (2010) 124905.

[7] E. Fill, et al., New Journal of Physics 8 (2006) 272.

[8] L. Veisz, et al., New Journal of Physics 9 (2007) 451.

[9] This research is supported by the Department of Energy (DE-FG52-09NA29415) and the National Science Foundation (DMR-0619573). J.A. Berger acknowledges the support of a Department of Education GAANN Fellowship. 